Monatsschr Kinderheilkd 2021 · 169:694-704 https://doi.org/10.1007/s00112-021-01230-9 Angenommen: 26. Mai 2021

Online publiziert: 2. Juli 2021

๑) Springer Medizin Verlag GmbH, ein Teil von Springer Nature 2021

\section{Redaktion}

H. Girschick, Würzburg

F. Zepp, Mainz

\title{
Künstliche Intelligenz bei Bildauswertung und Diagnosefindung
}

\section{Was bringt das in der Kinder- und Jugendradiologie?}

\author{
Hans-Joachim Mentzel \\ Sektion Kinderradiologie, Institut für Diagnostische und Interventionelle Radiologie, Universitätsklinikum \\ Jena, Jena, Deutschland
}

\section{Zusammenfassung}

\section{In diesem Beitrag}

- Standortbestimmung und Blick in die Zukunft

- Historie

- Tägliche Anwendungen in der Routine Kommunikation - Workflow-Management $•$ Bilderstellung und -verarbeitung • Klassifikation und Quantifizierung • Segmentierung • Detektion, Bewertung und Vorhersage - Radiomics - Gestaltung und Übermittlung radiologischer Befunde

- Ausblick

Aus Gründen der besseren Lesbarkeit wird in diesem Artikel überwiegend das generische Maskulinum verwendet. Dies impliziert immer beide Formen, schließt also die weibliche Form mitein.



QR-Code scannen \& Beitrag online lesen

Die Radiologie ist von stetem Wandel geprägt und definiert sich über den technologischen Fortschritt. Künstliche Intelligenz (KI) wird die praktische Tätigkeit in der Kinder- und Jugendradiologie künftig in allen Belangen verändern. Bildakquisition, Befunderkennung und -segmentierung sowie die Erkennung von Gewebeeigenschaften und deren Kombination mit Big Data werden die Haupteinsatzgebiete in der Radiologie sein. Höhere Effektivität, Beschleunigung von Untersuchung und Befundung sowie Kosteneinsparung sind mit der Anwendung von $\mathrm{KI}$ verbundene Erwartungshaltungen. Ein verbessertes Patientenmanagement, Arbeitserleichterungen für medizinisch-technische Radiologieassistenten und Kinderund Jugendradiologen sowie schnellere Untersuchungs- und Befundzeiten markieren die Meilensteine der Kl-Entwicklung in der Radiologie. Von der Terminkommunikation und Gerätesteuerung bis zu Therapieempfehlung und -monitoring wird der Alltag durch Elemente der KI verändert. Kinder- und Jugendradiologen müssen daher grundlegend über KI informiert sein und mit Datenwissenschaftlern bei der Etablierung und Anwendung von KI-Elementen zusammenarbeiten.

Schlüsselwörter

Maschinenlernen · Computer assistierte Diagnose · "Convolutional neural network" · Big Data Kinderradiologie

Die Digitalisierung verändert Umwelt und Gesellschaft und so auch das Gesundheitswesen. Die diagnostische Radiologie ist von einer analogen Technik nahezu komplett in die digitale Welt, bestehend aus Nullen und Einsen, transformiert. Dieses Fachgebiet ist prädestiniert für den nächsten Entwicklungsschritt - die Anwendung künstlicher Intelligenz (KI) in der Anfertigung und Auswertung digitaler Bilddaten.

Wird KI Radiologen ersetzen? Ein Szenario, das manche befürchten, das aber nach Expertensicht nicht eintreffen wird. Allerdings werden die Kollegen scheitern, die künftig den Einsatz von Verfahren der $\mathrm{KI}$ ablehnen. Eine Vorhersage, wie die radiologische Welt in 10 Jahren aus- sieht, ist nicht möglich. Fakt ist schon jetzt, das KI die Arbeit verbessern wird Terminmanagement, Untersuchungsplanung, Durchführung, Befunderstellung und -kommunikation, Qualitätsmanagement ([26]; - Abb. 1). In all diesen Bereichen wird künftig mit Anwendungen aus der KI zusammengearbeitet werden. Radiologen können sich bei sinnvollem Einsatz der KI auf komplexe Fälle und die klinische Arbeit fokussieren und intensiv(er) der Kommunikation mit Patienten und Kollegen widmen. Die künftige Ausbildung der Studierenden und die Weiterbildung in der Radiologie müssen daher die Begrifflichkeiten und Grundlagen zu $\mathrm{KI}$ und zum Umgang mit den Algorithmen und ihrer kritischen Anwendung sowie 
Hier steht eine Anzeige.

黑 Springer 
die entsprechende Reflexion beinhalten (- Tab. 1)

\section{Standortbestimmung und Blick in die Zukunft}

Tagtäglich werden in radiologischen $\mathrm{Ab}$ teilungen und Instituten Hunderttausende von Bildern erzeugt. Allein eine einzige Ganzkörper-MRT-Untersuchung besteht aus bis zu 6000 Bildern, eine Polytrauma-Spiral-CT ebenfalls aus mehreren Tausend Bildern. Radiologen benötigen für deren Auswertung sehr viel Zeit und höchste Konzentration; nichts soll übersehen werden - neben Hauptbefunden gibt es Neben- und Zufallsbefunde, die evtl. in

\section{Abkürzungen}

ACR American College of

RadiologyAIAmerican College of Radiology "Artificial intelligence"

ASIR "Adaptive statistical iterative reconstruction"

AWMF Arbeitsgemeinschaft der Wissenschaftlichen Medizinischen Fachgesellschaften

$B M D \quad$ "Bone mineral density"

CAD "Computer assisted diagnosis"

CAKUT "Congenital anomalies of the kidney and urinary tract"

CNN "Convolutional neural network"

COVID-19 "Coronavirus disease 2019"

CT Computertomographie

DICOM "Digital imaging and communication in medicine"

$D L \quad$ "Deep learning"

DXR "Digital X-ray radiogrammetry" (digitale Radiogrammetrie)

HIV "Human immunodeficiency virus"

JPEG Joint Photographic Experts Group

KI Künstliche Intelligenz

KIS Krankenhausinformationssystem

$\mathrm{MCl} \quad$ Metakarpalindex

mIP "Minimum intensity projection"

MIP "Maximum intensity projection"

ML "Machine learning"

MPR "Multiplanar reconstruction"

MRT Magnetresonanztomographie

MW Mittelwert

PACS „Picture archiving and communication system"

PERCH Pneumonia Etiology Research in Child Health

PI Porositätsindex

PIMS „Pediatric inflammatory multisystem syndrome"

RIS Radiologie Informations System

ROC "Receiver operating characteristics"

SD Standardabweichung

SSK Strahlenschutzkommission

UBO "Unidentified bright object"
Zukunft eine krankmachende Bedeutung für den Patienten haben können. Dieser Aspekt ist insbesondere bei Kindern zu berücksichtigen, die ihr ganzes Leben noch vor sich haben und künftige, sich entwickelnde pathologische Veränderungen noch erleben werden. Umso größer ist die Erwartungshaltung in der Kinder- und Jugendradiologie gegenüber künftiger Unterstützung durch Verfahren der KI.

\section{॥) Künftig werden KI-Elemente neben Diagnose und Prognose auch Therapievorschläge unterbreiten können}

Computerprogramme suchen schon seit Jahren als "Computer-assisted diagnosis"(CAD)-Systeme hypothesenbasiert nach Auffälligkeiten in radiologischen Bildern und markieren diese. Ein Hauptanwendungsgebiet dieser Technik in der Kinder- und Jugendradiologie ist die Analyse von Karporadiogrammen zur Bestimmung des Skelettalters. In Zukunft werden Elemente der $\mathrm{Kl}$ selbstständig Algorithmen entwickeln, die die individuellen Bildinformationen mit anamnestisch klinischen Angaben des jeweiligen Patienten und Big Data aus großen Netzwerken kombinieren und neben Diagnose und Prognose auch eine geeignete Behandlung vorschlagen. "Machine learning" (ML) als eine Form von $\mathrm{Kl}$ ist in der Lage, eigenständig und ohne explizites Programmieren anhand gesammelter Daten Muster zu erkennen - ohne Ermüdung wird an Bildprozessierung und -interpretation gearbeitet [21]. Der Faktor Mensch wird aber trotz dieser Selbstständigkeit der Technik unverändert in der ethischen Verantwortung sein, diese Informationen zu sichten und ihre Vertrauenswürdigkeit kritisch zu prüfen, bevor er sie nutzt. Dies gilt insbesondere, da für eine Zulassung von KI-Software in Deutschland bislang lediglich eine einfache CE-Kennzeichnung ausreichend ist, also keine randomisierten Studien notwendig sind, um die Effektivität und Sicherheit der KI nachzuweisen. So kann auch der Software ein Fehler unterlaufen; die Haftung liegt dann aber sicherlich nicht beim Entwickler der Software, bzw. die KI kann nicht haftbar gemacht werden.

\section{Historie}

Bereits für die ersten Computer in den 1950er-Jahren wurde der Begriff „artificial intelligence" (AI) geprägt. In den 1980erJahren wurden erste Professuren zur KIErforschung geschaffen. Der Einsatz von computergestützter Auswertesoftware in der diagnostischen Radiologie wird in den 2000er-Jahren als CAD-Systeme in der Detektion von Herdbefunden in der Lunge und in der Mamma etabliert. In der Kinderradiologie hielten erste Anwendungen zur automatisierten Skelettalterbestimmung Einzug in die Routine (z.B. BoneXpert ${ }^{\mathrm{TM}}$, Visiana, Hørsholm, Dänemark, [23]). Die Kombination der Vermessung von knöchernen Strukturen der Mittelhand und der Analyse der Porosität des Knochens gestattet zudem eine Abschätzung der Knochendichte [19]. Sehr häufig werden bei Kindern Röntgenaufnahmen des Thorax angefertigt. Ihre Beurteilung ist komplex und subjektiv, sodass mehrere Radiologen je nach Erfahrung, Training, Müdigkeit und Fokussierung zu teilweise sehr verschiedenen Bewertungen kommen. Die CAD-Systeme können digitale Röntgenbilder automatisch analysieren, ermüden nicht und liefern ohne Intraobserver- und Interobserver-Variabilität einen Befund. Im Rahmen der Studie Pneumonia Etiology Research in Child Health (PERCH) wurde CAD4Kids entwickelt, um auf Thoraxröntgenaufnahmen Pneumonien entsprechend den WHOKriterien zu detektieren („dense or fluffy opacity that occupies either a portion, lobe or entire lung, with or without air bronchograms", [18]), und konnte eine Sensitivität von $76 \%$ und eine Spezifität von $80 \%$ im Vergleich zu 3 erfahrenen Radiologen erreichen. Automatische Segmentierung, Texturanalyse, Scoring und die Einbeziehung klinischer Angaben (z.B. „Humanimmunodeficiency-virus"[HIV]-Status) gehörten zu den trainierten Schritten dieses Algorithmus. Ein wesentlicher Vorteil dieser Studie war der große Datenumfang (858 Kinder), allerdings wurde lediglich ein JPEG-Format ausgewertet. Der Einfluss der entsprechend reduzierten Datenqualität (256 Graustufen, Formatkompression) könnte durch die Analyse von DICOMDaten vermindert werden. 


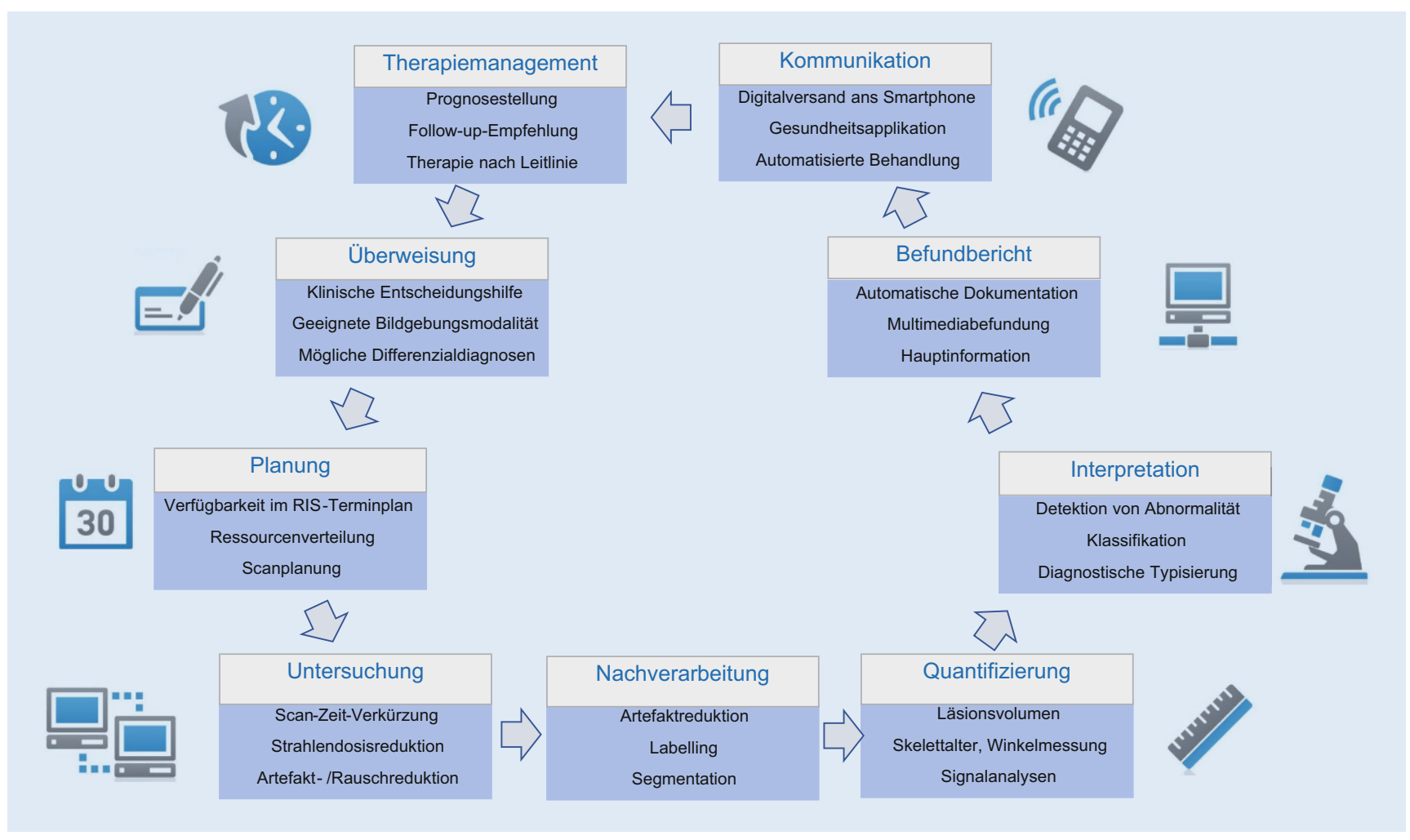

Abb. 1 A Patientenweg von der Überweisung bis zu radiologischem Befundbericht und Therapieplanung mit nachfolgenden Kontrollen mithilfe der Bildgebung. Übersicht, wie künstliche Intelligenzwerkzeuge die klinische Praxis in der Kinderradiologie verbessern können. RIS Radiologie Informations System. (Teile der Abbildung [Icons] wurden erstellt mit freundl. Genehmigung $\odot$ Erhan Ergin/Fotolia, alle Rechte vorbehalten)

Durch Steigerung der Rechenleistung moderner Computersysteme mit Einführung von "graphics processing units" sowie die Entwicklung von Algorithmen gelang die Etablierung von KI und ML. Künstliche Intelligenz lernt hypothesenfrei unter Zuhilfenahme von Bilddaten - z. B. aller Sequenzen bei einer MRT-Untersuchung -, klinischen Parametern, Laborwerten und genetischen Informationen. Die Güte von KI-Anwendungen hängt von der Datenqualität ab, mit denen das System trainiert wird [8]. Beim ML werden das überwachte (basiert auf durch Experten vorbereiteten Datensätzen, bei denen In- und Output definiert sind) und das unüberwachte (freie) Lernen unterschieden [7]. Beim freien Lernen wird der Algorithmus trainiert, selbstständig Merkmale in den zur Verfügung gestellten Daten zu identifizieren und anhand dieser Gruppen zu unterscheiden. Hierfür ist eine wesentlich größere Datenmenge notwendig. Deep learning als eine Form des ML beruht auf der Anwendung künstlicher neuronaler Netze („,convolutio- nal neural network", CNN), die gemeinsam interagieren, um ein Problem zu lösen [4].

\section{॥) Künstliche Intelligenz lernt hypothesenfrei}

Auch wenn in der Radiologie mittlerweile Fragen zu Kl-Anwendungen tagtäglich präsent sind, spielt das Thema in der Kinder- und Jugendradiologie noch keine so große Rolle. Dies ist einerseits der wesentlich größeren Arbeitslast in der Erwachsenenradiologie mit einem gigantischen Bilddatenaufkommen und dem groBen Wunsch nach Entlastung bei der Routinebefundung geschuldet, andererseits ist es durch den Mangel an ausreichend großen Trainingsdatensätzen in der Kinderradiologie erklärbar. Das betrifft sowohl die Quantität als auch die Heterogenität der Daten in der Kinder- und Jugendmedizin. Wesentlich ist der Anspruch auf personalisierte Medizin in der Kinderradiologie, was dazu führt, dass eine Vielzahl der Untersuchungen individuell geplant und durchgeführt wird.
Bislang werden $\mathrm{Kl}$-Anwendungen, abgesehen von der in radiologischen Praxen und Abteilungen etablierten Spracherkennung verschiedenster Anbieter, nahezu ausschließlich an universitären Zentren entwickelt und evaluiert. Ursache sind hierzulande fehlende bzw. unzureichende Infrastrukturen in den Gesundheitseinrichtungen, falsche Erwartungen und unklare Gesetzeslagen. Es ist daher nicht verwunderlich, das sich auch die KI-Forschung eher in anderen Ländern entwickelt. In globalen Wettkämpfen unter Wissenschaftlern werden mittlerweile für bestimmte definierte Fragestellungen KI-basierte Algorithmen entwickelt, wie z. B. zur Lungenrundherddetektion. Die Radiological Society of North America führt jährlich eine KI-Challenge durch. Im Jahr 2019 widmete sich dieser Wettkampf der Vorhersage des Knochenalters [10]. Es wurden 14.236 Röntgenaufnahmen der Hand zur Verfügung gestellt, wobei 12.611 zum Trainieren der Algorithmen, 1425 zur Validierung und 200 Karporadiogramme zum vergleichenden Test genutzt wurden. Es 
Tab. 1 Kurzgefasste Begriffsdefinitionen zur Arbeit mit Werkzeugen der künstlichen Intelligenz. (Nach Moore et al. [21])

\begin{tabular}{|c|c|}
\hline Term & Definition \\
\hline $\begin{array}{l}\text { Artificial } \\
\text { intelligence }\end{array}$ & $\begin{array}{l}\text { Wissenschaftszweig zur Erforschung von Computern und -programmen, die } \\
\text { Aufgaben ausführen, die normalerweise menschliche Intelligenz erfordern }\end{array}$ \\
\hline $\begin{array}{l}\text { Machine } \\
\text { learning }\end{array}$ & $\begin{array}{l}\text { Teilgebiet der künstlichen Intelligenz, in dem Algorithmen so trainiert werden, } \\
\text { dass sie Aufgaben ausführen. Basis ist das Lernen aus Mustern und nicht eine } \\
\text { explizite Programmierung }\end{array}$ \\
\hline $\begin{array}{l}\text { Deep learn- } \\
\text { ing }\end{array}$ & $\begin{array}{l}\text { Möglichkeit des maschinellen Lernens, bei der der Algorithmus selbst die besten } \\
\text { Funktionen zum Klassifizieren bereitgestellter Daten erarbeitet }\end{array}$ \\
\hline $\begin{array}{l}\text { Supervised } \\
\text { learning }\end{array}$ & $\begin{array}{l}\text { Trainingsmethode maschinellen Lernens, bei der jedes Beispiel im Datensatz } \\
\text { einer bestimmten Kategorie zugeordnet wird }\end{array}$ \\
\hline $\begin{array}{l}\text { Unsupervised } \\
\text { learning }\end{array}$ & $\begin{array}{l}\text { Trainingsmethode des maschinellen Lernens, bei der die Datenbeispiele nicht } \\
\text { kategorisiert werden, sondern das Modell selbst anhand inhärenter Merkmale } \\
\text { eine Gruppenzuordnung vornimmt }\end{array}$ \\
\hline $\begin{array}{l}\text { Convolutional } \\
\text { network }\end{array}$ & Häufig verwendete Art des maschinellen Lernens mit Netzwerken \\
\hline Labeling & Zuordnung jedes Beispiels zu einer bestimmten Kategorie \\
\hline Classification & $\begin{array}{l}\text { Vorhersage der Kategorie einer Läsion (Merkmal) anhand des Gesamtbildes oder } \\
\text { einer bestimmten Region }\end{array}$ \\
\hline Detection & Vorhersage der Lokalisation potenzieller Läsionen oder bestimmter Merkmale \\
\hline Segmentation & $\begin{array}{l}\text { Aufteilung der Daten in sinnvolle Teildaten durch Identifikation von Pixeln oder } \\
\text { Voxeln bestimmter Merkmalskategorie }\end{array}$ \\
\hline
\end{tabular}

wurden 105 Verfahren auf Basis neuronaler Netze eingereicht. Die besten Resultate ergaben eine Abweichung von 4,2 bis 4,5 Monaten vom Referenzstandard; ein Wert, den sehr erfahrene Kinderradiologen im Vergleich kaum erreichen.

\section{Tägliche Anwendungen in der Routine}

Die Themen Kommunikation, WorkflowManagement von Untersuchungen, Bildverarbeitung, Segmentierung und Detektion, Bewertung und "radiomics" zählen zu den Anwendungsgebieten von $\mathrm{KI}$ in der Radiologie. Die Verbreitung von KIAnwendungen und ihre Integration in den Arbeitsprozess erfolgen individuell in der radiologischen Abteilung, über Teleradiologieverbünde oder aber über AppStores. Diverse Großgerätehersteller entwickeln derzeit entsprechende Verbreitungsstrukturen wie beispielsweise Siemens Healthineers mit dem in Syngo.Via integrierten App-Store [11]. Start-ups wie MedTech Startup Deepc aus München bieten verschiedenste Software-Lösungen zu Identifikation, Charakterisierung und Quantifizierung von Läsionen, die in Zusammenarbeit mit Radiologen sorgfältig validiert werden müssen.

\section{Kommunikation}

In der Kommunikation können schon heute über Spracherkennung Chatbots („,artifical conversational entity") im Dialog mit den Patienten deren Symptome erfassen [28] und basierend auf "Deeplearning"-(DL)-Algorithmen künftig aus den geschilderten Problemen die geeignete Bildgebungsmodalität anhand vorhandener evidenzbasierter Vorgaben auswählen. Digitale Anmeldungen in der radiologischen Praxis oder in der Klinik, verknüpft mit dem Krankenhausinformationssystem (KIS), könnten die zeitraubende Terminvergabe übernehmen. Strukturierte Anforderungstexte werden durch $\mathrm{KI}$ analysiert, und entsprechend der Fragestellung und gültiger Leitlinien wird das geeignete bildgebende Verfahren vorgeschlagen [6]. Dies setzt allerdings voraus, dass die Zuweiser diese Texte im Vorfeld korrekt bzw. in ausreichender Weise ausfüllen und bestenfalls eine digitale Datenkartei des Kindes vorliegt. Neben den Leitlinien der Arbeitsgemeinschaft der Wissenschaftlichen Medizinischen Fachgesellschaften (AWMF) sind für die Auswahl die nationalen Orientierungshilfen der Strahlenschutzkommission (SSK) und auch internationale Angebote zu Rechtfertigung und Optimierung der Bild- gebung wie Image Gently und Image Wisely zu berücksichtigen, die in den Vorgaben des American College of Radiology (ACR) beschrieben sind und stetig aktualisiert werden [12].

\section{Workflow-Management}

Durch geeignete Software (z. B. Syngo Virtual $\mathrm{Cockpit}^{\circledR}$, Siemens Healthineers) können schon heute Großgeräteuntersuchungen wie z.B. ein MRT aus der Ferne gesteuert werden. Den Körpermaßen des Patienten entsprechend werden die Untersuchung vorbereitet, die Orientierung angepasst und geeignete Sequenzfolgen in Abhängigkeit von der Fragestellung automatisiert gestartet. Standardisierte Rekonstruktionstechniken (z. B. multiplanare Rekonstruktionen [MPR], Maximum- und Minimumintensitätsprojektionen [MIP, mIP] sowie dreidimensionale Darstellungen von Profil, Knochen, Gefäßen oder Organen) ohne Bearbeitung der Rohdaten werden erstellt. Die zunehmend knapper werdende Zahl medizinisch-technischer Radiologieassistenten (künftig Technologen für Radiologie) kann so effizienter eingesetzt werden.

\section{॥) Kl-gesteuertes zentrales Workflow-Management bietet Zeitersparnis und differenzierende Befundberichte}

Unternehmen stellen KI-Algorithmen (z. B. IntelliSpace AI Workflow Suite ${ }^{\circledR}$, Fa. Philips; Edison Open Al Orchestrator ${ }^{\circledR}$, Fa. GE) zur Verfügung, die das zentrale Workflow-Management steuern. Klinische Daten werden unmittelbar an die Modalität und die $\mathrm{KI}-$ Anwendung geliefert und ermöglichen die Bilddatenerstellung und -verarbeitung nach Dringlichkeit und ohne Eingriff des radiologischen Personals. Bei den Befundungslisten wird durch KI-Anwendungen eine Priorisierung vorgenommen, die basierend auf klinischen Angaben und Fragestellung sowie automatisch in den Bilddaten detektierter pathologischer Veränderungen (z.B. Pneumothorax) entscheidet, welche Untersuchung zuerst zu befunden ist [30]. Der Report wird künftig zunehmend standardisiert und strukturiert gestaltet [25], sodass Befundassistenten (z. B. Mint Medical) ihn vorbereiten und ak- 
Hier steht eine Anzeige.

黑 Springer 


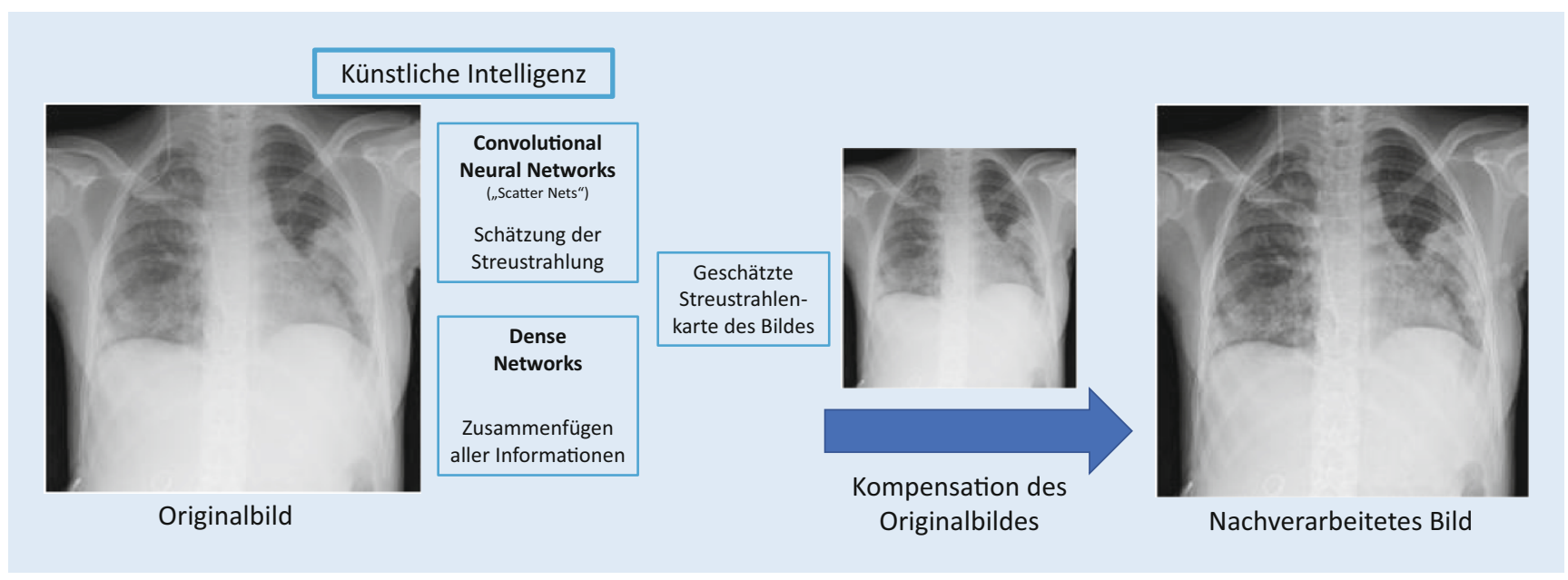

Abb. 2 \ Darstellung der Bildprozessierung unter Nutzung eines virtuellen Rasters (SimGrid, S-VueTM, Fa. Samsung). (Mit freundl. Genehmigung, @ K. Ebeling, alle Rechte vorbehalten)

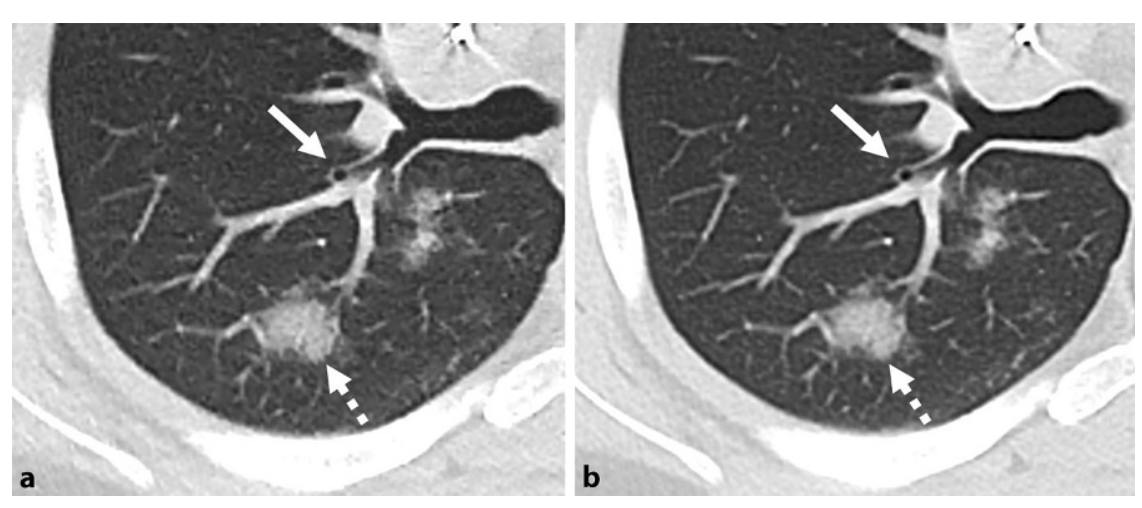

Abb. 3 ॥ Thorax-CT-Aufnahmen eines am „pediatric inflammatory multisystem syndrome“ erkrankten 15-Jährigen. Darstellung im Lungenfenster, Akquisition 0,625 mm Schichtdicke , Ultra-low-dose“Protokoll. a 2,5 mm dicke Rekonstruktion mit adaptiver statistischer iterativer Rekonstruktion (ASIR); durch den Einsatz von iterativer Rekonstruktion zur Dosiseinsparung resultiert ein höheres Bildrauschen. b 2,5 mm dicke Rekonstruktion mit ASIRund DL-Algorithmus. Durch den Einsatz von DL-Algorithmen resultiert trotz ASIR ein Bild mit schärferen Konturen und geringerem Bildrauschen

tuelle Untersuchungsbefunde bereits mit Voruntersuchungen im Verlauf markieren und segmentieren können. Die resultierende Zeitersparnis dürfte zur Akzeptanz der (bislang noch etwas unbeliebten) strukturierten Befundung führen. Strukturierte Befunde sind letztlich in der weiteren standardisierten Betreuung der Patienten sinnvoll. Zudem bieten DL-basierte Algorithmen wie z.B. AUTOStroke solution ${ }^{\circledR}$ (Fa. Canon Medical System) beim akuten Schlaganfall in Kombination mit der selbstständigen Bildakquisition und -analyse bereits einen zwischen ischämischen und hämorrhagischen Infarkten differenzierenden Befundbericht, der dem SchlaganfallNetzwerk zur Verfügung gestellt werden kann. Basierend auf 10.000 Fällen wurde der zugrunde liegende DL-Algorithmus für die Analyse und Differenzierung intrakranieller Blutungen trainiert.

\section{Bilderstellung und -verarbeitung}

Anwendungen der KI zu Bilderstellung und -verarbeitung haben sich mittlerweile in diologie etabliert. In der konventionellen Radiographie werden verschiedene Algorithmen eingesetzt, um die Bildqualität zu verbessern. So liefern virtuelle Raster bei bettseitig angefertigten Röntgenaufnahmen von Thorax und Abdomen eine wesentlich schärfere Abbildung bei reduzierter Strahlenexposition. Generell für die Untersuchung am Röntgenstativarbeitsplatz (Thoraxröntgen im Stehen) zur Reduktion der Streustrahlenunschärfe entwickelt, den verschiedenen Modalitäten der Ra- können sie bei Kindern auf der Intensivstation ab einem Körpergewicht von ca. $10 \mathrm{~kg}$ zu höherer Bildqualität ohne vermehrte Strahlenexposition genutzt werden. Der nach Leitlinien der Fachgesellschaften empfohlene Einsatz von physikalischen Rastern zur Streustrahlenreduktion ist mit einem höheren Dosisbedarf verbunden.

Beispielhaft ist dies in $\mathbf{A b b} .2$ dargestellt. Deep-learning-basierte Algorithmen tragen individuelle Patientendaten (Gewicht, Sagittaldurchmesser) über "convolutional neural" und "dense network" mit großen Datenmengen zusammen und schätzen den zu erwartenden Streustrahlenanteil im Bild ab. Anschließend wird das Bild über neuronale Netze (54 Lagen, 30.000 Bilder), die an anthropometrischen Modellen und klinischen Röntgenbildern trainiert wurden, analysiert und mit der abgeschätzten Streustrahlungskarte kompensiert, wodurch ein detailreiches schärferes Bild resultiert.

In der Computertomographie lassen iterative Rekonstruktionstechniken der Rohdaten und DL-Algorithmen eine weitere Dosisreduktion zu und liefern rauschärmere, kontrastreichere Bilder ([32]; - Abb. 3). Im MRT haben KI-Anwendungen das Potenzial zur Reduktion der Scan-Zeit [15], was insbesondere im Kindesalter zur größeren Akzeptanz der Untersuchung führt bzw. Untersuchungen in Sedierung ermöglicht und so die Zahl erforderlicher Narkose-MRT und damit verbundener Risiken vermindert. Andere 


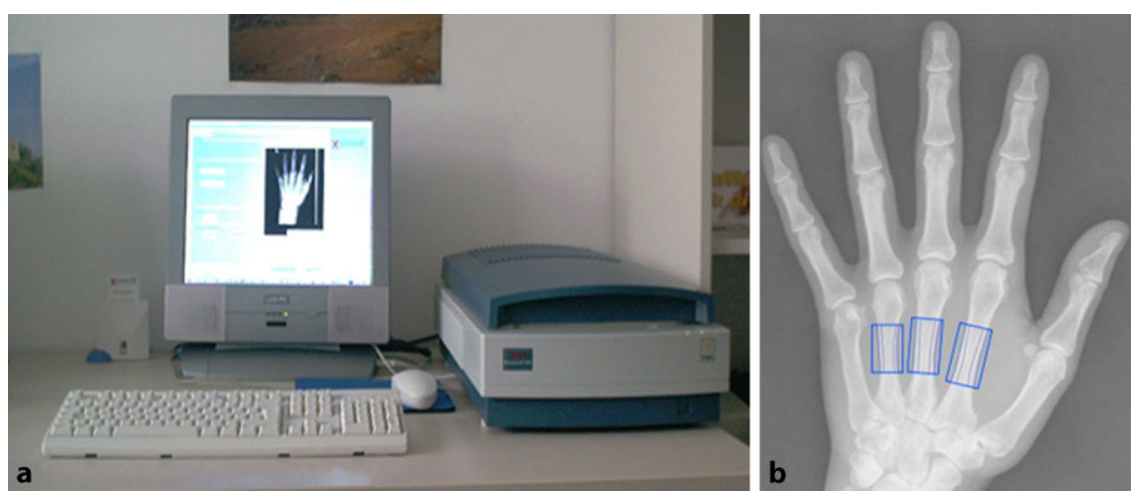

Abb. $4 \Delta$ Abschätzung der Knochenmineraldichte mithilfe der digitalen Radiogrammetrie. a ScannerEinheit (Pronosco X-posure System, V2. Sectra, Schweden) zur Digitalisierung der Röntgenaufnahme und zur automatisierten Kalkulation von Metakarpal-und Porositätsindex, b automatisierte Detektion und Markierung der Kortexstrukturen der Ossa metacarpalia II-IV, Bestimmung von kortikaler Dicke und Markraumdurchmesser. Unter Zuhilfenahme von CAD-Algorithmen Segmentierung und Texturanalyse. (In Anlehnung an Mentzel et al. [20])

DL-Verfahren gestatten eine Reduktion der i.v.-Applikation gadoliniumhaltiger Kontrastmittel in der MRT, deren Anwendung bei Kindern und die mögliche Ablagerung im Körper zu Diskussion und Verunsicherung bei den Eltern geführt hat [9]. Bei der Nachverarbeitung können störende Bildartefakte reduziert werden, wie am Beispiel der Diffusionstensorbildgebung der Wirbelsäule gezeigt werden konnte [1]. Eine automatisierte Kategorisierung und Kennzeichnung von Bilddaten (Labeling) bei Versand ins "picture archiving and communication system" (PACS) ist im Umgang mit den Bilddatensätzen - insbesondere bei onkologischen Patienten mit einer Vielzahl von Verlaufskontrollen bei der Befundung und Demonstration in interdisziplinären Fallbesprechungen sehr hilfreich.

\section{Klassifikation und Quantifizierung}

Algorithmen zur Klassifikation und zur Quantifizierung verbessern die Möglichkeiten bei Messverfahren in der Orthopädie (z.B. Cobb-Winkel bei Skoliose, Reimers-Index bei Hüftdysplasien). Wie bei der oben genannten Entwicklung von CAD-Systemen war für die Pädiatrie aufgrund der gewaltigen Datenmenge an standardisiert angefertigten Röntgenaufnahmen der Hand die Skelettaltersbestimmung für die Entwicklung eines entsprechenden Algorithmus prädestiniert [17]. Es gelang, ein neuronales Netz zu erstellen, das eine dem Experten äquivalente

\section{Detektion, Bewertung und} Vorhersage

Neben der Detektion durch fest programmierte CAD-Systeme als Basis kann beispielsweise ein an einer Vielzahl von Lungen-CT-Daten trainierter KI-Algorithmus (XGBoost) helfen, eine Bewertung und Vorhersage zu treffen, welcher Herdbefund sich künftig zu einem Malignom entwickeln wird. Lakhani und Sundaram haben einen Algorithmus zur Detektion von Lungentuberkulose mit einer exzellenten Effizienz und Genauigkeit entwickelt („Area under curve "[AUC]-Wert 0,99; [16]). Für die Lungen-CT bei interstitiellen Erkrankungen können auf ML basierende KI-Algorithmen objektiv und rasch Aussagen treffen, welche pathologische Veränderung vorliegt und deren Ausmaß quantifizieren [22]. Bereits heute kommerziell verfügbare Anwendungen wie Computer Aided Lung Informatics for Pathology Evaluation and Rating (CALIPER ${ }^{\circledR}$ ) stufen das Lungengewebe auf Voxelebene anhand von Dichte und Textur in verschiedene Klassen ein und weisen bei interstitiellen Lungenerkrankungen Erwachsener eine höhere Korrelation zur Lungenfunktion auf als die herkömmliche visuelle Analyse der Radiologen. Zur Befundung von Thoraxröntgenaufnahmen existieren Algorithmen, die bis zu 70 oder 90 pathologische Veränderungen erkennen, allerdings passen sie sich bislang schlecht in den radiologischen Workflow ein [8]. dienen zur Verlaufsbeurteilung von Marklagerveränderungen im Hirn (z.B. „unidentified bright objects" [UBO] bei Phakomatosen, Plaques bei multipler Sklerose) bzw. zum Therapiemonitoring bei Lungenherden [21]. Die automatisierte Segmentierung von Bilddatensätzen zur Volumetrie von Klein- und Großhirn mit Differenzierung von Liquorräumen, grauer und weißer Substanz sowie das Aufzeigen von Normabweichungen gestatten Kl-Assistenten. Die Volumenentwicklung der Liquorräume kann in den radiologischen Befundbericht automatisch integriert werden und ist beispielsweise für Therapieentscheidungen bei Kindern mit Hydrozephalus wichtig.

\section{॥) Für Erwachsene etablierte KI- Anwendungen müssen eigens für Kinder und Jugendliche trainiert werden}

Im Erwachsenenalter etablierte KI-Anwendungen dürfen nicht kritiklos und unreflektiert für Patienten im Kindesalter übernommen werden. So wies eine KI zur Frakturdetektion bei Erwachsenen eine Sensitivität von $98 \%$ und eine Spezifität von $99 \%$ auf, bei Kindern allerdings eine hohe falsch-negative Rate mit einer Sensitivität von $26-36 \%$. Die Spezifität von $95-98 \%$ war vergleichsweise sehr hoch [2]. Abhilfe könnte lediglich ein Training der KI an entsprechenden Datensätzen von Kindern und Jugendlichen schaffen. Bisher 
Tab. 2 Mittelwerte und Standardabweichungen der DXR-Parameter für Jungen. (Aus Mentzel et al. [20])

\begin{tabular}{|l|l|l|l|l|l|l|l|}
\hline $\begin{array}{l}\text { Alter } \\
\text { (Jahre) }\end{array}$ & $n$ & \multicolumn{2}{l}{ BMD } & MCI & PI \\
\cline { 3 - 8 } & & MW & \pm SD & MW & \pm SD & MW & \pm SD \\
\hline $6-8$ & 8 & 0,40 & $\pm 0,04$ & 0,36 & $\pm 0,05$ & 5,78 & $\pm 3,02$ \\
\hline $9-10$ & 17 & 0,41 & $\pm 0,03$ & 0,33 & $\pm 0,03$ & 5,28 & $\pm 2,29$ \\
\hline $11-12$ & 28 & 0,43 & $\pm 0,04$ & 0,35 & $\pm 0,04$ & 5,09 & $\pm 2,52$ \\
\hline $13-14$ & 32 & 0,48 & $\pm 0,05$ & 0,36 & $\pm 0,05$ & 5,48 & $\pm 2,56$ \\
\hline $15-16$ & 32 & 0,59 & $\pm 0,07$ & 0,45 & $\pm 0,06$ & 5,07 & $\pm 2,65$ \\
\hline $17-18$ & 35 & 0,62 & $\pm 0,05$ & 0,47 & $\pm 0,08$ & 4,69 & $\pm 2,11$ \\
\hline
\end{tabular}

$B M D$ „bone mineral density" $\left[\mathrm{g} / \mathrm{cm}^{2}\right], D X R$ "digital X-ray radiogrammetry" (digitale Radiogrammetrie), MCI Metakarpalindex, PI Porositätsindex, MW Mittelwert SD Standardabweichung

Tab. 3 Mittelwerte und Standardabweichungen der DXR-Parameter für Mädchen. (Aus Mentzel et al. [20])

\begin{tabular}{|l|l|l|l|l|l|l|l|}
\hline $\begin{array}{l}\text { Alter } \\
\text { (Jahre) }\end{array}$ & $n$ & BMD & & MCI & & PI & \\
\cline { 3 - 8 } & & MW & \pm SD & MW & \pm SD & MW & \pm SD \\
\hline $6-8$ & 12 & 0,39 & $\pm 0,03$ & 0,34 & $\pm 0,03$ & 4,58 & $\pm 1,37$ \\
\hline $9-10$ & 12 & 0,41 & $\pm 0,04$ & 0,37 & $\pm 0,04$ & 5,37 & $\pm 2,09$ \\
\hline $11-12$ & 19 & 0,46 & $\pm 0,04$ & 0,40 & $\pm 0,05$ & 3,52 & $\pm 1,74$ \\
\hline $13-14$ & 17 & 0,50 & $\pm 0,04$ & 0,42 & $\pm 0,05$ & 3,92 & $\pm 2,19$ \\
\hline $15-16$ & 15 & 0,55 & $\pm 0,04$ & 0,46 & $\pm 0,05$ & 4,11 & $\pm 2,62$ \\
\hline $17-18$ & 15 & 0,55 & $\pm 0,04$ & 0,49 & $\pm 0,08$ & 5,12 & $\pm 2,09$ \\
\hline
\end{tabular}

$B M D$ „bone mineral density“ $\left[\mathrm{g} / \mathrm{cm}^{2}\right], D X R$ "digital X-ray radiogrammetry" (digitale Radiogrammetrie), MCI Metakarpalindex, PI Porositätsindex, MW Mittelwert, SD Standardabweichung

evaluierte Anwendungen im Kindesalter sind beispielhaft die Al-Anwendung zur Frakturdetektion auf Röntgenbildern [27], zur Beurteilung von Knochenmarkbefunden bei nichtbakterieller Osteitis auf MRTBildern [3] oder die Klassifikation von angeborenen Anomalien von Nieren und ableitenden Harnwegen („,congenital anomalies of the kidney and urinary tract", CAKUT) anhand von Ultraschallbildern [33]. In der Kinderneuroradiologie konnten für die 3TMR-Spektroskopie Algorithmen zur Differenzierung von Medulloblastom, Ependymom und pilozytischem Astrozytom entwickelt werden; Subgruppen des Medulloblastoms können durch die Kombination von Texturanalyse, klinischen Biomarkern und Bildmerkmalen ohne Biopsie unterschieden werden [14].

\section{\) Radiomics ermöglicht}

Vorhersagen zu Tumorhistologie, -genetik, -metastasierung und Therapieansprechen

Ein wesentliches Problem der Anwendung von DL-Systemen ist die Fokussierung auf meist nur eine pathologische Verände- rung - andere Auffälligkeiten werden nicht erkannt, sodass es zu fatalen Fehleinschätzungen kommen kann. Als Beispiel werden Metastasen der Nebennieren benannt, die von der auf Lungenherde geschulten $\mathrm{KI}$ nicht erkannt werden. Verbessert werden kann dies durch das Schalten mehrerer parallel arbeitender neuronaler Netze in einer Anwendung [11].

\section{Radiomics}

Das Kunstwort Radiomics (auch „radiogenomics") ist eine Zusammensetzung aus Radiologie und Genetik und bezeichnet die Möglichkeit, aus individuell erzeugten quantitativen Bilddaten unter Nutzung großer medizinischer Datenbanken statistisch basierte Aussagen zu Entität und Prognose zu erstellen. Neben Statistik (Korrelation mit klinischen Endpunkten) wird $M L(D L)$ bei der automatischen Extraktion quantitativer Merkmale aus den Bilddaten eingesetzt. Aktuell wird diese Möglichkeit digitaler Bildanalyse bei der Charakterisierung und Differenzierung von Pneumonien, die mit der "coronavirus disease 2019" (COVID-19) assoziiert sind, erfolgreich an- gewandt [13]. Seit den ersten Publikationen zu Radiomics 2012 konnte das Potenzial zur Vorhersage von Tumorhistologie und -genetik, Metastasierung und Therapieansprechen weiterentwickelt werden. Im Kindesalter wurden Daten zu Osteound Weichteilsarkom publiziert [5, 31].

In einem Multizenterprojekt wurden klinische Daten und Ergebnisse der Bildgebung bei Kindern mit neonataler hypoxisch-ischämischer Enzephalopathie gesammelt. Eine $\mathrm{KI}$ mit ML soll an diesem Datenpool Algorithmen entwickeln, die Aussagen zur Prognose gestatten [29]. Ergebnisse der Bildgebung werden in AlAnwendungen integriert, die Biomarker für bestimmte neurokognitive Erkrankungen identifizieren, um diese möglichst frühzeitig zu diagnostizieren und eine entsprechende (Früh-)Förderung bzw. Therapie zu ermöglichen [6].

\section{Gestaltung und Übermittlung radiologischer Befunde}

Bei der Gestaltung und Übermittlung der radiologischen Befunde werden Kl-Algorithmen eingesetzt, um in den mitunter sehr ausführlichen Beschreibungen und Beurteilungen die wesentlichen Fakten zu identifizieren und zu markieren. Algorithmen in der Spracherkennung werden entwickelt, um besonders bedeutsame Befunde (z.B. Fehllage des Trachealtubus, Spannungspneumothorax, Hirnödem mit drohender Einklemmung) hervorzuheben und den Kliniker entsprechend darüber zu informieren. Im Fall von seltenen Erkrankungen oder schwierigen Differenzialdiagnosen könnte Software helfen, eine befundbasierte Literaturrecherche selbstständig durchzuführen und wesentliche Publikationen zum Fall herauszufiltern [24].

\section{Ausblick}

Künstliche Intelligenz und ML werden in der Kinder- und Jugendradiologie künftig als wertvolles Instrument zur Bildanalyse verfügbar sein und die tägliche Arbeit von der Terminierung und Planung bis zu Durchführung und Integration des Untersuchungsergebnisse in den Behandlungsplan beeinflussen. Kinder- und Jugendradiologen sind gefordert, gewissen- 
haft und sorgsam mit diesen Innovationen umzugehen und sie kritisch zu hinterfragen. Bei Erwachsenen bereits etablierte KIAnwendungen können nicht unreflektiert ins Kindes- und Jugendalter übernommen werden. Der individuelle Patientendatenschutz und ethische Fragen werden bei dem in großem Umfang zunehmendem Bild- und Datenversand und der Bearbeitung durch internationale Netzwerkstrukturen verstärkt zu beachten sein. Die Sicherheit in der Interaktion von Patientenidentifikations- und Patientendatenkarten mit Datenbanken der ambulanten und stationären Versorgung sowie der Krankenkassen ist zu prüfen. Gesetzliche Vorgaben sind entsprechend dem Nutzen der Anwendung von $\mathrm{KI}$ und $\mathrm{ML}$ in der Patientenversorgung und deren Risiken permanent zu entwickeln. Eine Möglichkeit zur Vergütung des Einsatzes von KI-Anwendungen wird erforderlich sein, da sowohl die Implementierung als auch die Pflege und Weiterentwicklung von $\mathrm{KI}$-Elementen kostenintensiv sind.

\section{Fazit für die Praxis}

- Künstliche Intelligenz (KI) und Maschinenlernen (ML) sind vielversprechend zur Erleichterung der Arbeit in der Kinder- und Jugendradiologie einsetzber.

- KI kann perspektivisch die Befundsicherheit erhöhen

- Ethische Ansprüche und Datenschutzfragen sind nicht abschließend geklärt.

- Eine kritische Überprüfung der Ergebnisse von $\mathrm{KI}$-Anwendungen ist notwendig. Expertise über die eingesetzten Algorithmen und hohe Qualität der Trainingsdaten (Big Data) sind die Grundvoraussetzungen für einen effektiven Einsatz von KI.

- Durch automatisierte Bildauswertung kann Expertenwissen mit prädiktiven Biomarkern verschiedener Kategorien kombiniert werden und Aussagen zu Prognose und geeigneter Therapie ermöglichen.

- Die Nutzung standortübergreifender Datensätze in Forschungsverbünden der Kinder- und Jugendradiologie ist mit erheblichem Ressourcenaufwand verbunden und erfordert neben besserer Standardisierung der radiologischen Untersuchungen auch ein Vergütungsmodell.

\section{Korrespondenzadresse}

\section{Prof. Hans-Joachim Mentzel}

Sektion Kinderradiologie, Institut für Diagnostische und Interventionelle Radiologie, Universitätsklinikum Jena

Am Klinikum 1, 07747 Jena, Deutschland

hans-joachim.mentzel@med.uni-jena.de

\section{Einhaltung ethischer Richtlinien}

Interessenkonflikt. H.-J. Mentzel gibt an, dass kein Interessenkonflikt besteht.

Für diesen Beitrag wurden vom Autor keine Studien an Menschen oder Tieren durchgeführt. Für die aufgeführten Studien gelten die jeweils dort angegebenen ethischen Richtlinien.

\section{Literatur}

1. Alizadeh M, Conklin CJ, Middleton DM, Shah P, SaksenaS, Krisa Letal (2018) Identification of ghost artifact using texture analysis in pediatric spinal cord diffusion tensor images. Magn Reson Imaging 47:7-15

2. Alqahtani FF, Messina F, Offiah AC (2019) Are semitautomated software program designed for adults accurate for the identification of vertebral fractures in children? Eur Radiol 29:6780-6789

Hier steht eine Anzeige. 
3. Bhat CS, Chopra M, Andronikou S, Paul S, WernerFligner Z, Merkoulovitch A et al (2020) Artificial intelligence for interpretation of segments of whole body MRI in CNO: pilot study comparing radiologists versus machine learning algorithm. Pediatr Rheumatol 18:47

4. Chartrand G, Cheng PM, Vorontsov E, Drozdzal M, Turcotte S, Pal CJ, Kadoury S, Tang A (2017) Deep learning: a primer for radiologists. Radiographics 37:2113-2131. https://doi.org/10.1148/rg. 2017170077

5. Chen X, Huang Y, He L, Zhang T, Zhang L, Ding H (2020) CT-based radiomics to differentiate pelvic rhabdomyosarcoma from yolk sac tumors in children. Front Oncol. https://doi.org/10.3389/ fonc.2020.584272

6. Davendralignam N, Sebire NJ, Arthurs OJ, Shelmerdine SC (2021) Artificial intelligence in paediatric radiology: future opportunities. BJR 94:111720200975. https://doi.org/10.1259/bjr. 20200975

7. Erickson BJ, Korfiatis P, Akkus Z, Kline TL (2017) Machine learning for medical imaging. Radiographics 37:505-515. https://doi.org/10.1148/rg. 2017160130

8. Forsting M (2019) Artificial intelligence with radiology as a trailblazer for super-diagnostic: an essay. Fortschr Röntgenstr 191:73-78. https://doi. org/10.1055/a-0808-7772

9. Gong E, Pauly JM, Wintermark M, Zaharchuk G (2018) Deep learning enables reduced gadolinium dose for contrast-enhanced MRI. J Magn Reson Imaging 48:330-340

10. Halabi SS, Prevedello LM, Kalpathy-Cramer J, Mamonov AB, Bilbily A, Cicero M, Pan I, Pereira LA Sous RT, Abdala N, Kitamura FC, Todberg HH, Chen L, Shih G, Andriole K, Kohli MD, Erickson BJ, Flanders AE (2019) The RSNA pediatric bone age machine learning challenge. Radiology 290:498-503. https://doi.org/10.1148/radiol. 2018180736

11. Haubold J (2020) Künstliche Intelligenz in der Radiologie. Was ist in den nächsten Jahren zu erwarten. Radiologe 60:64-69. https://doi.org/10. 1007/s00117-019-00621-0

12. Hernanz-Schulman M, Frsh DP, Bettman MA (2021) Appropriate use criteria and computerized decision support for the pediatric population: background, pediatric rapid response committee and future direction. Pediatr Radiol 51:371-377

13. Huang Y, Zhang Z, Liu S, Li X, Yang Y, Ma J, Li Z, Zhou J, Jiang Y, He B (2021) CT-based radiomics combined with signs: a valuable tool to help radiologist discriminate COVID-19 and influenza pneumonia. BMC Med Imaging. https://doi.org/ 10.1186/s12880-021-00564-w

14. Iv M, Zhou M, Shpanskaya K, Perreault S, Wang Z, Tranvinh E et al (2019) Imaging based radiomic signatures of distinct molecular subroups of medulloblastoma. AJNR Am J Neuroradiol 40:154-161

15. Johnson PM, Recht MP, Knoll F (2020) Improing the speed of MRI with artificial intelligence. Semin Muskuloskelet Radiol 24:12-20. https://doi.org/ $10.1055 / \mathrm{s}-0039-3400265$

16. Lakhani P, Sundaram B (2017) Deep learning at chest radiography: automated classification of pulmonary tuberculosis by using convolutional neural networks. Radiology 284:574-582

17. Larson DB, Chen MC, Lungren MP, Halabi SS, Stence NV, Langlotz CP (2018) Performance of a deep-learning neural networkmodel in assessing skeletal maturity on pediatric hand radiographs. Radiology 287:313-322

\section{Artificial intelligence in image evaluation and diagnosis. What is the benefit for pediatric radiology?}

Radiology is characterized by constant change and is defined by technological progress Artificial intelligence (Al) will change all aspects of practical work in pediatric radiology in the future. Image acquisition, diagnosis recognition and segmentation as well as the recognition of tissue properties and their combination with big data will be the main areas of application in radiology. Higher effectiveness, acceleration of investigations and making the diagnosis as well as cost savings are expectations associated with the use of Al. Improved patient management, easier work for radiology assistants and pediatric radiologists as well as faster examination and diagnosis times mark the milestones of Al development in radiology. From appointment communication and device control to treatment recommendations and monitoring, the daily life will be changed by elements of Al. Pediatric radiologists must therefore be informed about the basics of $\mathrm{Al}$ and work together with data scientists in establishing and using $\mathrm{Al}$ elements.

\section{Keywords}

Machine learning · Computer assisted diagnosis · Convolutional neural network · Big data · Pediatric radiology

18. Mahomed N, van Ginneken B, Philipsen RHHM, Melendez J, Moore DP, Moodley H, Sewchuran T, Mathew D, Madhi SA (2020) Computer-aided diagnosis for World Health Organization-defined chest radiograph primary-endpoint pneumonia in children. Pediatr Radiol 50:482-449

19. Mentzel HJ, John U, Boettcher J, Malich A, Pfeil A, Vollandt R, Misselwitz J, Kaiser WA (2005) Evaluation of bone-mineral density by digital X-ray radiogrammetry (DXR) in pediatric renal transplant recipients. Pediatr Radiol 35:489-494

20. Mentzel HJ, Malich A, Kunze C, Kramer A, Pfeil A Boettcher J, Spielmann RP, Kaiser WA (2007) Digitale Radiogrammetrie (DXR). Referenzwerte der Knochenmineraldichte für Kinder und Jugendliche. Monatsschr Kinderheilkd 155:16-22

21. Moore MM, Slonimsky E, Long AD, Sze RW, lyer RS (2019) Machine learning concepts, concerns and opportunitites for a pediatric radiologist. Pediatr Radiol 49:509-516

22. Prayer F, Röhrich S, Pan J, Hofmanninger J Langs G, Prosch H (2020) Künstliche Intelligenz in der Bildgebung der Lunge. Radiologe 60:42-47. https://doi.org/10.1007/s00117-019-00611-2

23. van Rijn R, Lequin MH, Thodberg $H H$ (2009) Automatic determination of Greulich and Pyle bone age in healthy Dutch children. Pediatr Radiol 39:591-597

24. Schoeb D, Suarez-Ibarrola R, Hein S, Dressler FF, Adams F, Schlager D et al (2020) Use of artificial intelligence for medical literature search: random ized controlled trial using the Hackathon format. Interact J Med Res 9:e16606

25. Shea LAG, Towbin AJ (2019) The state of structured reporting: the nuance of standardized language. Pediatr Radiol 49:500-508. https://doi.org/10. 1007/s00247/-019-04345-0

26. Summers RM (2018) Deep learning lends a hand to pediatric radiology. Radiology 287:323-325. https://doi.org/10.1148/radiol.2018172898

27. Thian YL, Li Y, Jagmohan P, Sia D, Chan VEY, Tan RT (2019) Convolutional neural networks for automated fracture detection and localization on wrist radiographs. Radiology 1:e180001
28. Tudor Car L, Dhinagaran DA, Kyaw BM, Kowatsch T, JotyS, Theng YL et al (2020) Conversational agents in health care: scoping review and conceptual analysis. J Med Internet Res 22:e17158

29. Weiss RJ, Bates SV, Song Y, Zhang Y, Herzberg EM, Chen YC, Song Y et al (2019) Mining multi-site clinical data to develop machine learning MRI biomarkers: application to neonatal hypoxicischemic encephalopathy. J Transl Med 17:385

30. Winkel DJ, Heye T, Weitkert TJ et al (2019) Evaluation of an ai-based detection software for acute findings in abdominal computed tomography scans: toward an automated work list prioritization of routine $\mathrm{CT}$ examinations. Invest Radiol 54:55

31. Wu Y, Xu L, Yang P, Lin N, Huang X, Pan W, Li H, Lin P, Li B, Bunpetch V, Luo C, Jiang Y, Yang D, Huang $M$, Niu T, Ye Z (2018) Survival prediction in highgrade osteosarcoma using radiomics of diagnostic computed tomography. EBioMedicine 34:27-34

32. Zhang H, Wang J, Zeng D, Tao X, Ma J (2018) Regularization strategies in statistical image reconstruction of low-dose X-ray CT: a review. Med Phys 45:e886-e907. https://doi.org/10.1002/mp. 13123

33. Zheng Q, Furth SL, Tasian GE, Fany Y (2019) Computer-aided diagnosis of congenital abnormalities of the kidney and urinary tract in children based on ultrasound imaging data by integrating texture image features and deep transfer learning image features. JPediatr Urol 15:75.e1-75.e7 